Bryan Wiltgen, Swaroop Vattam, Michael Helms, Ashok Goel \& Jeannette Yen. Learning Functional Models of Biological Systems for Biologically Inspired Design. In Proc. 11th IEEE International Conference on Advanced Learning Technologies, Athens, Georgia, USA, July 2011.

http://ieeexplore. ieee.org/xpl/articleDetails.jsp?arnumber=5992342

\title{
Learning Functional Models of Biological Systems for Biologically Inspired Design
}

\author{
Bryan Wiltgen ${ }^{1}$, Swaroop Vattam, ${ }^{1}$ \\ Michael Helms ${ }^{1,2}$ \\ ${ }^{1}$ Design \& Intelligence Laboratory \\ Georgia Institute of Technology \\ Atlanta, GA, USA \\ \{bwiltgen, svattam,mhelms3\}@cc.gatech.edu
}

\author{
Ashok K. Goel ${ }^{1,2}$, Jeannette Yen ${ }^{2}$ \\ ${ }^{2}$ Center for Biologically Inspired Design \\ Georgia Institute of Technology \\ Atlanta, GA, USA \\ \{goel\}@cc.gatech.edu, \\ jeannette.yen@biology.gatech.edu
}

\begin{abstract}
Biologically inspired design uses cross-domain analogies from biology to engineering to enhance design creativity and innovation. This analogical transfer requires conceptual understanding of biological systems. In this paper, we describe a prototype interactive knowledge-based design environment called DANE for supporting conceptual understanding through learning about functional models of biological designs. We present initial results from deploying DANE in an interdisciplinary class on biologically inspired design, indicating that designers found DANE's functional models useful for conceptualizing complex systems.
\end{abstract}

Keywords- Biomimetic Design, Biological Systems, Functional Models, Model Learning.

\section{INTRODUCTION}

Biologically inspired design (or biomimetic design) uses analogies to biological systems to foster creativity and derive innovative solutions to design problems [1,2]. Design analogies from biology to engineering require conceptual understanding of biological systems from a design perspective. Since 2005 Georgia Tech's Center for Biologically Inspired Design has offered a senior-level, project-based interdisciplinary undergraduate course on biologically inspired design (ME/ISyE/MSE/PTFe/BIOL 4740) [3]. Teaching and learning biologically inspired design offers several challenges because despite its many successes, the practice of biologically inspired design is largely ad hoc, with little systemization of biological knowledge from the perspective of design. The question then becomes how can we represent knowledge of biological systems to support conceptual understanding of their designs? In this paper, we describe a prototype interactive learning environment called DANE for supporting conceptual understanding of biological systems through learning about functional models of their designs.

\section{DESIGN BY ANALOGY TO NATURE ENGINE}

The Design by Analogy to Nature Engine (DANE) interactively aids designers in finding and understanding biological systems that might be relevant to a given engineering design problem, so that they may potentially extract, abstract, and transfer the appropriate biological knowledge to their design problem. Herbert Simon in the cognitive sciences [4] and Pahl \& Beitz [5] in engineering, among many others, have emphasized the centrality of functions in design knowledge and processing. In past work, we have built libraries of engineering designs indexed by their functions [6] and represented as Structure-BehaviorFunctions models [7]. An SBF model of a complex natural or technological system (1) explicitly specifies the structure, functions, and behaviors (i.e., the causal processes that result in the functions) of the system, (2) uses functions as indices to organize knowledge of behaviors, (3) annotates state transitions in a behavior with causal explanation for it, (4) organizes the knowledge in $\mathrm{F} \rightarrow \mathrm{B} \rightarrow \mathrm{F} \rightarrow \mathrm{B} \rightarrow \ldots \rightarrow \mathrm{F}(\mathrm{S})$ abstraction hierarchy, and (5) provides an ontology for representing its structures, functions and behaviors. DANE also enables designers to construct and refine StructureBehavior-Function (SBF) models of biological and engineering systems [8]. In other work on learning about complex ecological systems in middle school science, we have found that construction of SBF models enables students to acquire conceptual understanding of the systems [9].

When we introduced DANE into the biologically inspired design classroom, the library contained about forty SBF models, including twenty-two "complete" models of biological systems and subsystems. The remaining were either SBF models of engineering systems or only partial models of biological systems. Biological systems in DANE are at several levels of scale from the sub-cellular to organ function to organism sub-system (e.g. locomotion) levels.

Systems are indexed by system-function pairs and retrieved by function name (e.g., "flamingo filter-feeds self"), by subject (e.g., "flamingo"), and/or by verb (e.g., "filter-feeds"). Upon selecting a system-function pair, users are presented with a multi-modal representation. A system can be represented in text descriptions and images, as well as through visualizations of behavior and structure models.

Behavior and structure models are themselves represented as directed graphs, which may be annotated with text descriptions and images. The nodes and edges represent either structural elements and connections (for structure models) or states and transitions (for behavior models), respectively. We provide an example of a partial behavior model, this time for the system "kidney filters blood," in Figure 1. This "kidney filters blood" partial behavior model describes the movement of blood through the kidney through increasingly smaller vessels until the blood arrives at the nephron. Note that our figure only shows a few states and transitions of the total behavior. 


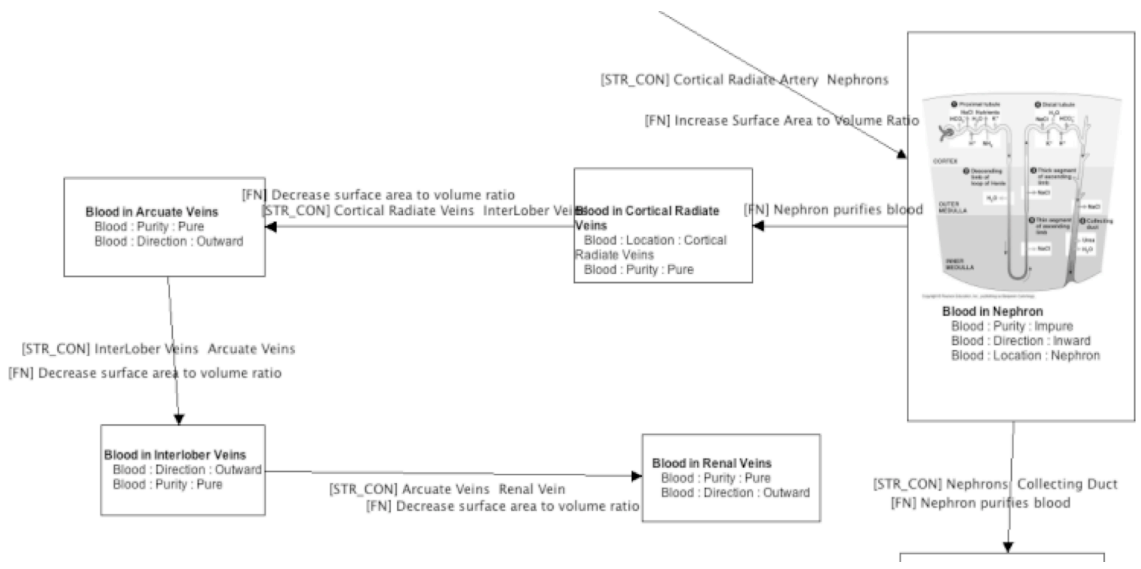

Fig. 1. Partial Behavior Model of "Kidney Filters Blood" in DANE.

The SBF representation schema places an emphasis on the causal relationships within each system and makes explicit the function/sub-function relationships between systems [6]. We hypothesize that presenting systems in this manner will help biologists and engineers identify systems that are relevant to their design problem and transfer their understanding to a design solution. For example, an engineer might scan models in DANE until he/she comes across a system that has a similar initial and objective state to his/her design problem. Then, by inspecting the structure and behavior of that system, the engineer might formulate a technological solution that implements a similar set of structures and causal transitions.

\section{APPLICATION CONTEXT}

We deployed DANE in the Fall 2009 semester session of ME/ISyE/MSE/PTFe/BIOL 4740, a project-based, seniorlevel, undergraduate course taught by biology and engineering faculty affiliated with Georgia Tech's Center for Biologically Inspired Design [3]. The class composition was interdisciplinary, comprised of students from biology, mechanical engineering, and a variety of other disciplines.

The course was broken up into three components: lectures, found object exercises, and a semester-long biologically inspired design team project. For the project, teams of 4-6 students were formed such that each team had at least one biology student and students from different schools of engineering. Each team was given a broad problem in the domain of dynamic, adaptable, sustainable housing such as heating or energy use.

The class is taught without any technological aids for design or research. Students are encouraged to perform their own research on biological systems through resources such as Google Scholar (scholar.google.com), Encyclopedia of Life (www.eol.org), Web of Science (www.wokinfo.com), and Ask Nature (www.asknature.org). While these sources contain quality information, they often return results that are difficult to use.

\section{TRAINING AND DEPLOYMENT}

At the end of the third week of the class, we introduced our tool through an hour long tutorial session presented by the authors during class-time [7]. Students were already comfortable with the idea of biologically inspired design, grouped in their semester design teams, and aware of their semester-long project. Each team had brought at least one computer from which the DANE tool was accessible.

The point of our lesson was to motivate DANE, acquaint students with the kind of representations that exist within it, and teach them how to interact with it.

\section{RESUlTS}

We collected several kinds of data during the deployment. In this paper, we report on an exit interview we conducted with one student and our analysis of course reflections done by each student.

After the semester ended, we conducted a 14-question interview about DANE with a student from the class. Although we recognize that a single student is not a sufficient sample for how the entire class felt about our tool, we felt this student could provide valuable feedback about the tool because she alone had posted questions in a troubleshooting forum about the software. The interview was taped and then transcribed with permission of the interviewee. We present the highlights.

When asked how she would rate the importance of DANE to her semester-long project on a scale from 1 to 10 with 10 being of vital importance, the student gave a rating of 5, stating "it wasn't extremely, crucially vital, but it wasn't something that was not necessary" and "in the end we could've probably done without it, but I think it helped us to conceptualize." When probed about what she meant by "conceptualize," the student responded, "I mean, like, conceptualize, like, I think in boxes. Only because I'm in industrial engineering so I think in a lot of - I mean they look like flow charts. So that's what I like about DANE so I could, like...build a flow chart, essentially. From, like, the beginning stage to the end stage of a process."

Not all responses were positive. When asked if DANE improved her understanding of biological systems, the student said no because, "I wasn't looking up information. I was trying to input information into the database."

Finally, when asked if she would recommend that other students use DANE, she answered yes, stating it's a "good 
resource" for "trying to build the analogies. And for like visualizing the connections, like the different properties. Like when my team first looked at it our overall function was regulate, and from regulate we had like regulate water, regulate energy, regulate heat, and you could just like break that up and you could go into DANE and see which- like we all independently like came up with objects in nature that had these properties and see if they were tied to each other."

Students were asked by the instructor to write a final paper that reflected upon their experiences in the class. 36 such reflection papers were submitted. In six of those, DANE was mentioned by name. In two papers, both written by engineering students, the comments were explicitly positive (e.g., "I thought that DANE was a very useful tool to help decompose our system into its parts" and "A resource database (DANE!) would be VERY helpful in this class."). In another paper, also written by an engineering student, the comments were explicitly negative (e.g., "DANE did not really help in our communication" and "it had good intentions, but I did not feel that it had great potential as an aide."). The remaining three papers contained neutral statements. Three of the six reflections mentioned DANE as a research repository, two described it as a modeling tool, and one described it in terms of aiding communication.

\section{CHALLENGES}

Based on the observed results of our deployment, we have drawn several lessons. The first is that students were not willing to invest the time and effort to build models. Unfortunately, without a sufficient number of models, students are unlikely to find DANE useful as a reference resource. However, at $40-100$ hours per model, a small group of isolated researchers has little hope of creating sufficient breadth for general usability, so encouraging student model-building is a challenge we must overcome.

Students did appear to value DANE as a means to (a) organize their understanding of systems, and (b) test their own ability to represent a design case. In our student interview, the student mentions that DANE was a useful tool for conceptualizing systems and in making analogies.

Although DANE only explicitly appeared in one-sixth of the final reflections, we saw many different perspectives. Some students viewed it as a repository, some as a modeling/design environment, and at least one as a communication medium. However, based on the small number of students that mentioned DANE, it's apparent that we did not sufficiently convince the class of DANE's usefulness.

\section{CONCLUSIONS}

In this paper, we described an interactive learning environment - the Design by Analogy to Nature Engine (DANE) - for supporting biologically inspired design. We also described the deployment of DANE to help interdisciplinary design teams in performing an extended biologically inspired design project in a classroom setting. Although we struggled with motivating DANE's usage, DANE succeeded in the sense that the students were able to use DANE when they wanted and both the student we interviewed and two of the final project reflection journals said that DANE was a useful addition to their workflow.

On the other hand, we learned that DANE appears to support conceptual understanding of biological systems from a design perspective. In particular, it seems that the SBF knowledge representation scheme enables students to conceptualize functions of biological systems and to organize their knowledge of the systems around their functions. We know from past work that this kind of functional and causal understanding of complex systems is important for supporting design processes, These preliminary results appear promising enough to continue working on interactive learning environments for understanding biological systems in support of biologically inspired design.

\section{ACKNOWLEDGMENTS}

We acknowledge Georgia Tech's Center for Biologically Inspired Design for its support. In particular, we thank Professors David Hu, Craig Tovey and Marc Weissburg who helped Jeannette Yen teach the ME/ISyE/MSE/PTFe/BIOL 4740 class in Fall 2009. We also thank the students of the class including Jing Li. We are grateful to the US National Science Foundation that has generously supported this research through an NSF CreativeIT Grant (\#0855916) entitled Computational Tools for Enhancing Creativity in Biologically Inspired Engineering Design. A longer paper on DANE is available in [8].

\section{REFERENCES}

[1] Benyus, J. (1997) Biomimicry: Innovation Inspired by Nature. New York: William Morrow.

[2] Vincent, J., \& Mann, D. (2002) Systematic Transfer from Biology to Engineering. Philosophical Transactions of the Royal Society of London, 360: 159-173.

[3] Simon, H. A. (1996). The Sciences of the Artificial, $3^{\text {rd }}$ Edition, MIT Press.

[4] Pahl, G., \& Beitz. W. (1996) Engineering Design: A Systematic Approach. English edition edited by K. Wallace. $2^{\text {nd }}$ Edition. Springer.

[5] Yen, J., Helms, M., Vattam, S., \& Goel, A. (2010) Evaluating biological systems for their potential in engineering design. Advances in Natural Science 3(2): 27-40.

[6] Goel, A. (1992) Representation of Design Functions in ExperienceBased Design. In Intelligent Computer Aided Design, D. Brown, M Waldron, and H. Yoshikawa (editors), pp. 283-308, Amsterdam, Netherlands: North-Holland, 1992.

[7] Goel, A., Rugaber, S., \& Vattam, S. (2009) Structure, Behavior \& Function of Complex Systems: The Structure-Behavior-Function Modeling Language. AI for Engineering Design, Analysis and Manufacturing, 23: 23-35, 2009.

[8] Vattam, S., Wiltgen, B., Helms, M., Goel, A., \& Yen, J. (2010) DANE: Fostering Creativity in and through Biologically Inspired Design. In Proc. First International Conference on Design Creativity, Kobe, Japan, November 2010, pp. 127-132.

[9] Vattam, S., Goel, A., Rugaber, S., Hmelo-Silver, C., Jordan, R., Gray, S., \& Sinha, S. (2011) Understanding Complex Natural Systems by Articulating Structure-Behavior-Function Models. Educational Technology \& Society, 14(1): 66-81 